\title{
Think easy
}

\section{Manchmal sind es in der Praxis die einfachen Überlegungen, die dem Probanden rasch weiterhelfen.}

In dieser Rubrik präsentieren wir Ihnen interessante Fälle oder Fallstricke aus der Praxis. Wir laden Sie weiterhin recht herzlich ein, interessante und praxisrelevante Fälle zu präsentieren und ersuchen Sie um Übermittlung der dementsprechenden Manuskripte an: redaktion@sportmedizin.or.at Geben Sie Ihre Erfahrungen weiter und profitieren Sie von den Erfahrungen der Sport- und präventivmedizinischen Community!

\section{Fallstrick 1}

Es mag banal klingen, aber ein typisches Beispiel zeigt, wie Sportler von Geräten verunsichert werden:

Ein 26 jähriger Hobbyläufer trainiert auf den Marathon und stellt fest, dass sein Puls bei leichten Anstrengungen schon über 200/min. hinaufgeht. Beunruhigt sucht er mich auf und ich belaste ihn am Ergometer, um zu sehen, ob er unter Belastung eine Tachykardie entwickelt oder sich mein primärer Verdacht eines Gerätefehlers erhärtet. Er zeigt eine völlig normale Herzfrequenzund Blutdruckregulation und erreicht erst bei Höchstbelastung einen Puls von 204/min.

Sein Pulsabnehmer hatte einen Sensordefekt und wurde daraufhin ausgetauscht.

\section{Fallstrick 2}

Eine 38 jährige etwas adipöse Lehrerin kam zu mir, da sie durch Training und Ernährungsumstellung abnehmen wollte (!!!). Nach einer Durchuntersuchung einschließlich Labor bekam sie ein Trainingsprogramm, das sie sehr konsequent durchführte. Nach 6 Monaten war wieder eine Ergometrie vereinbart, um den Fortschritt zu sehen und das Training zu adaptieren. In den letzten Wochen bemerkte sie jedoch einen Leistungsabfall und Atemnot bei Belastung, - Symptome, die für sie zunächst nicht erklärbar waren. Die Trainingsanamnese ergab keinen Anhaltspunkt für ein Übertraining, es gab auch keine interkurrente Erkrankung.

Differentialdiagnostisch hatte ich neben einer ganzen Liste von Möglichkeiten sogar eine Pulmonalarterielle Hypertonie im Hinterkopf......da heißt es aber zuallererst an das Naheliegende denken: komplettes Blutbild, Serumeisen und Ferritin und siehe da, eine Eisenmangelanämie durch hohen menstruellen Blutverlust war des einfachen Rätsels Lösung! Eine 3-monatige orale Eisensubstitution verbesserte die Leistungsfähigkeit der Patienten in den erwarteten Bereich.

\section{Fazit für die Praxis}

Nicht zu kompliziert denken, wie auf einer Stufenleiter von der einfachen zur teuren Diagnostik!

\section{Fallstrick 3}

Eine 22 jährige sportliche Studentin wird mir von einem Kollegen zur Ergometrie und Lungenfunktion überwiesen, da sie angibt, sich in letzter Zeit beim Berggehen leistungsmäßig so schwach zu fühlen und so rasch außer Atem zu kommen.

Schon beim Hereingehen, fällt mir ihre auffallende Blässe auf. Anamnesti-
Auch bei sportlichen Personen muss eine adäquate Anamnese durchgeführt werden

sche Fragen nach starker Regelblutung, Medikamenteneinnahmen, vegetarischer Ernährung usw. verneint die Patientin. Auf eine Ergometrie wird vorerst verzichtet, um eine mögliche Anämie abzuklären. Tatsächlich findet sich eine massive Eisenmangelanämie (Ery 3,99T/l, Hb 5, 7 g/dl, Htk 23\%, MCV 58 fl, MCH 14,3 pg, MCHC 24,6 g/dl, Eisen 11 $\mu \mathrm{g} / \mathrm{dl}$, Ferritin $<5 \mathrm{ng} / \mathrm{ml}$ ), so dass am ehesten eine Eisenresorptionsstörung in Frage kommt.

Die Patientin wird an eine hämatologische Fachabteilung zur weiteren $\mathrm{Ab}$ klärung überwiesen.

\section{Fazit für die Praxis}

Nicht blind diagnostische Anforderungen erfüllen, sondern Augen auf, eine sorgfältige Anamnese machen und darnach handeln. Spart dem Gesundheitssystem oder dem Patienten Kosten und belastende Diagnostik.

\section{Zur Person}

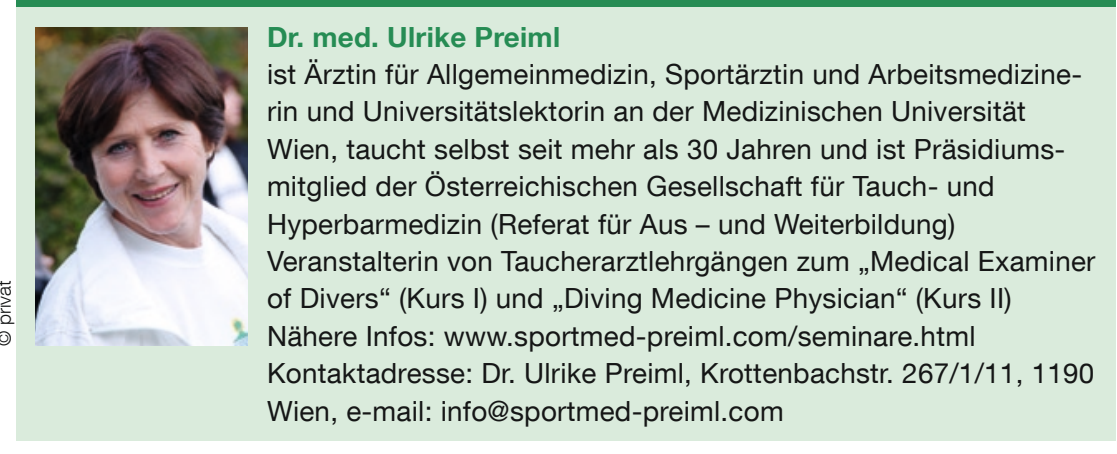

